\title{
NK cell cytotoxicity mediated by 2B4 and NTB-A is dependent on SAP acting downstream of receptor phosphorylation
}

\author{
Stephan Meinke ${ }^{1,2}$ and Carsten Watzl ${ }^{1,3 *}$ \\ 1 Institute for Immunology, University Heidelberg, Heidelberg, Germany \\ ${ }^{2}$ Center for Hematology and Regenerative Medicine, Karolinska Institutet, Stockholm, Sweden \\ ${ }^{3}$ Leibniz Research Centre for Working Environment and Human Factors—IfADo, Department for Immunology, Dortmund, Germany
}

\section{Edited by:}

Eric O. Long, National Institute of Allergy and Infectious Diseases, USA

\section{Reviewed by:}

Sumati Rajagopalan, National Institute of Allergy and Infectious Diseases, USA

Daniel McVicar, National Cancer Institute, USA

\section{*Correspondence:}

Carsten Watzl, Leibniz Research Centre for Working Environment and Human Factors-IfADo,

Ardeystrasse 67, D-44139

Dortmund, Germany.

e-mail: watz@ifado.de
2B4 (CD244) and NK-T-B-antigen (NTB-A, CD352) are activating receptors on human natural killer (NK) cells and belong to the family of signaling lymphocyte activation molecule (SLAM)-related receptors (SRR). Engagement of these receptors leads to phosphorylation of their cytoplasmic tails and recruitment of the adapter proteins SLAM-associated protein (SAP) and Ewing's sarcoma-activated transcript-2 (EAT-2). X-linked lymphoproliferative syndrome $(X L P)$ is a severe immunodeficiency that results from mutations in the SAP gene. 2B4 and NTB-A-mediated cytotoxicity are abrogated in XLP NK cells. To elucidate the molecular basis for this defect we analyzed early signaling events in SAP knockdown cells. Similar to XLP NK cells, knockdown of SAP in primary human NK cells leads to a reduction of 2B4 and NTB-A-mediated cytotoxicity. We found that early signaling events such as raft recruitment and receptor phosphorylation are not affected by the absence of SAP, indicating the defect in the absence of SAP is downstream of these events. In addition, knockdown of EAT-2 does not impair 2B4 or NTB-A-mediated cytotoxicity. Surprisingly, EAT-2 recruitment to both receptors is abrogated in the absence of SAP, revealing a novel cooperativity between these adapters.

Keywords: natural killer cells, human, cell activation, cell surface molecules, cytotoxicity

\section{INTRODUCTION}

Activation of natural killer (NK) cells is regulated by the balance of signals originating from activating and inhibitory receptors (Lanier, 2008). The receptors 2B4 (CD244) and NK-T-B-antigen (NTB-A, CD352) are activating receptors on NK cells that induce cytotoxicity and cytokine production. They belong to the family of signaling lymphocyte activation molecule (SLAM)-related receptors (SRR) (Claus et al., 2008). More and more evidence is emerging demonstrating that this family of Ig-like receptors plays a role in fine-tuning of immune responses, e.g., in germinal center formation or development of innate-like $\mathrm{T}$ cells (Schwartzberg et al., 2009). All SRR are homophilic with the exception of $2 \mathrm{~B} 4$ that binds to $\mathrm{CD} 48$, another Ig-like protein with broad expression in the hematopoietic system (Claus et al., 2008). SRR signaling is dependent on phosphorylation of their cytoplasmic domains at immunoreceptor tyrosine-based switch motifs (ITSM). In humans, phosphorylated ITSMs can recruit the small adapter proteins SLAM-associated protein (SAP) and Ewing's sarcoma-activated transcript-2 (EAT-2). These adapter molecules comprise only one Src homology 2 (SH2) domain and a short C-terminal extension (Ma et al., 2007; Claus et al., 2008). ITSM-bound SAP mediates signal transduction by recruiting the Src-family kinase FynT (Ma et al., 2007), which then phosphorylates downstream signaling molecules, e.g., Phospholipase C- $\gamma$ or Vav-1 (Watzl et al., 2000; Chen et al., 2004). Signal transduction molecules recruited by ITSM-bound EAT-2 have not been identified yet.

SAP plays an important role for lymphocyte function, as mutations in SAP result in a severe immunodeficiency called X-linked lymphoproliferative syndrome (XLP). Patients suffering from XLP show impaired humoral immune responses, defects in cytotoxic lymphocytes, and often develop a fatal mononucleosis after EBV infection (Ma et al., 2007). 2B4 and NTB-A-mediated cytotoxicity are defective in NK cells from XLP patients (Ma et al., 2007). One study demonstrated that $2 \mathrm{~B} 4$ engagement could even mediate inhibitory signals in XLP patients (Parolini et al., 2000). SAP ${ }^{-/-}$mice, which display similar immune defects like XLP patients, are used as an animal model for XLP (Ma et al., 2007).

Early signaling events after binding of 2B4 to its ligand CD48 are recruitment of the receptor to lipid rafts (Watzl and Long, 2003), phosphorylation of the four ITSM by Src-family kinases (Watzl et al., 2000; Watzl and Long, 2003), association of adapter molecules SAP and EAT-2 (Tangye et al., 1999; Morra et al., 2001), and recruitment of the Src-kinase FynT (Chen et al., 2004). Similarly, engagement of NTB-A leads to phosphorylation of the two ITSM and the recruitment of SAP and EAT-2 (Bottino et al., 2001; Eissmann and Watzl, 2006).

There are contradicting reports concerning the role of SAP and SAP-recruited FynT in these events. On the one hand, 
there are reports suggesting that $2 \mathrm{~B} 4$ phosphorylation takes place independently from SAP: pervanadate treatment of NK cells from XLP patients leads to 2B4 phosphorylation (Parolini et al., 2000), and antibody mediated cross-linking of 2B4 has recently been reported to induce receptor phosphorylation in NK cells from $\mathrm{SAP}^{-/-}$mice, as well as from mice lacking all SAP-family adaptors (Dong et al., 2012). In addition SAP cannot be found in association with the non-phosphorylated receptor in NK cells from healthy donors (Eissmann et al., 2005), which indicates that receptor phosphorylation precedes SAP-mediated FynT recruitment. On the other hand, there are reports suggesting that SAPmediated Fyn recruitment is necessary for 2B4 phosphorylation: one study used a transfected cell line, in which co-transfection with SAP, but not co-transfection with a non-FynT-binding mutant form allowed 2B4 phosphorylation after receptor crosslink (Chen et al., 2004). Another study analyzing NK cells from $\mathrm{SAP}^{-/-}$mice found impaired $2 \mathrm{~B} 4$ phosphorylation after receptor cross-link (Bloch-Queyrat et al., 2005). Furthermore, 2B4 has been shown to be a target of FynT-mediated phosphorylation (Eissmann et al., 2005).

In this study we wanted to investigate how SAP and EAT-2 contribute to the early signaling events after 2B4 and NTB-A engagement in order to gain further insight into the molecular basis for the defective signaling of 2B4 and NTB-A in XLP NK cells.

\section{RESULTS \\ KNOCKDOWN OF SAP LEADS TO REDUCED 2B4 OR NTB-A-MEDIATED CYTOTOXICITY}

To study the mechanisms underlying the reduced $2 \mathrm{~B} 4$ or NTB-A-mediated cytotoxicity observed in NK cells from XLP patients we investigated the effect of SAP or EAT-2 knockdown in primary human NK cells. Transfection of in vitro propagated, IL-2-activated NK cells with siRNAs against SAP, EAT-2, or both mRNAs led to a strong reduction in protein expression of about 90\% (Figure 1A). The expression level of the Src-family kinase FynT was not changed. Knockdown of SAP diminished 2B4- and NTB-A-mediated cytotoxicity (Figure 1B) similar to the defect that has been reported for NK cells from XLP patients (Ma et al., 2007). In contrast, knockdown of EAT-2 did not decrease cytotoxicity mediated by these two receptors. Furthermore, NK cells with a double knockdown showed no significant further reduction of cytotoxicity compared to NK cells with knockdown of SAP alone (Figure 1B). The observed effects were not due to general impairment of NK cytotoxicity by the knockdown, as cell lysis triggered by NKG2D engagement was similar for all transfected cells (Figure 1B). These findings confirm that the impaired cytotoxicity of XLP NK cells is caused by defective signaling after target cell contact and not due to defective NK cell development caused by the absence of SAP. The related EAT-2, which can mediate signaling pathways leading to cytotoxicity in association

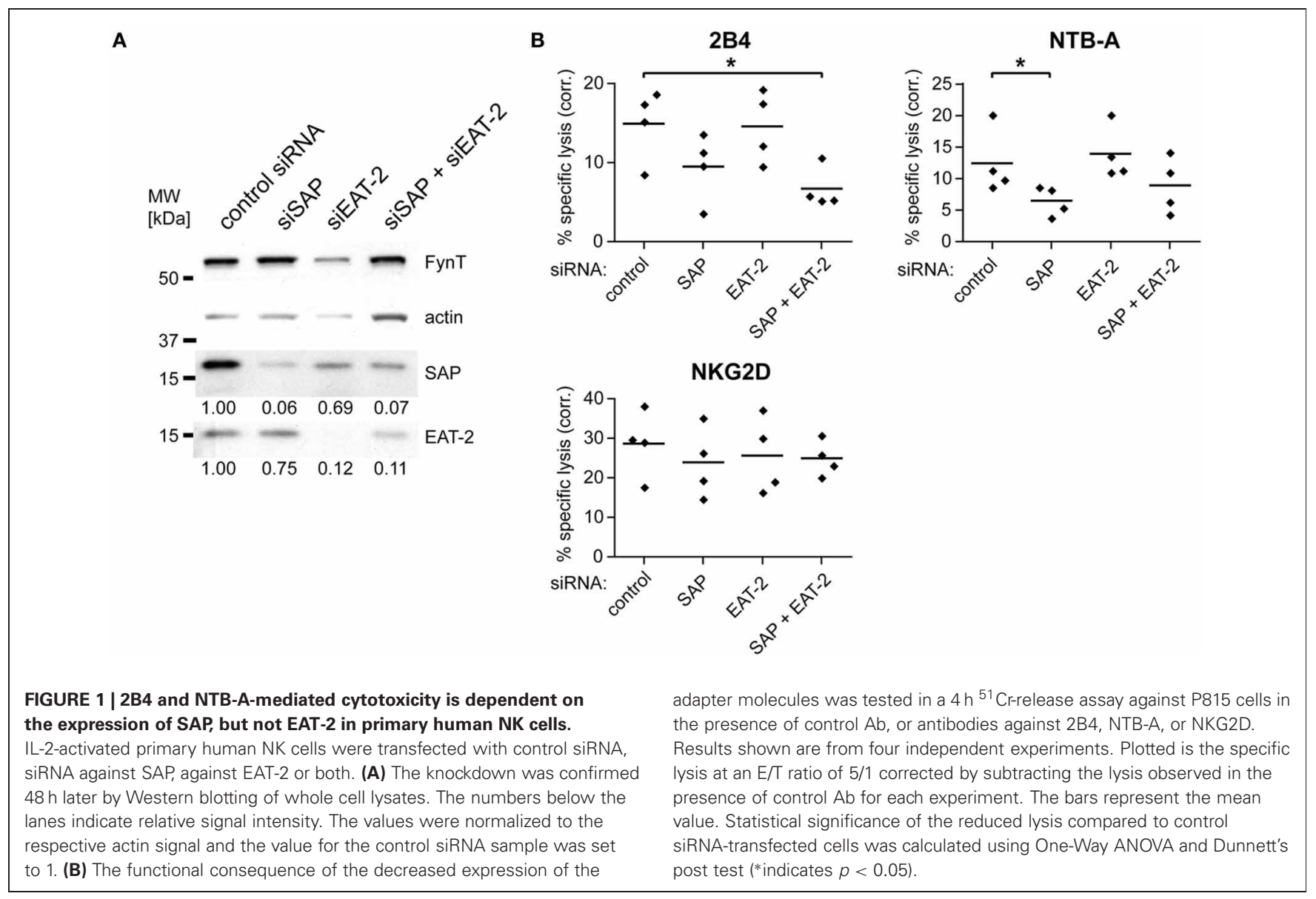


with the SRR CRACC (CD2-like receptor activating cytotoxic cells) (Bouchon et al., 2001), seems to regulate different pathways in 2B4 and NTB-A signaling, as its knockdown had no impact on cytotoxicity.

Because the numbers of primary NK cells recovered after transfection were too small for sufficient biochemical analysis of signaling events, we generated a stable knockdown of SAP by shRNA expression in the NK cell line NK92-C1. The SAP expression level in knockdown cells was about $10 \%$ of the amount expressed in control cells (Figure 2A). We also used two EAT-2 knockdown vectors, but the reduction of EAT-2 expression was only minor (data not shown). Similar to primary NK cells, NK92-C1 cells with SAP knockdown showed strongly diminished 2B4-mediated cell lysis. The already low cytotoxicity induced by NTB-A engagement in control cells was further reduced in SAP knockdown cells (Figure 2B). Like in primary cells cytotoxicity mediated by a SAP-independent receptor, NKp30, was not affected (Figure 2B). A similar, albeit weaker effect of SAP knockdown was observed in the NK-like cell line YTS (data not shown). As the knockdown had the same effect on cytotoxicity in the cell lines and the primary NK cells, we used the knockdown cell lines to analyze the molecular basis of this signaling defect.

\section{RECRUITMENT OF 2B4 TO LIPID RAFTS IS INDEPENDENT OF SAP}

One of the first events in 2B4 signaling is recruitment of the receptor to lipid rafts (Watzl and Long, 2003). Therefore, we investigated whether this early signaling event is impaired in the absence of SAP. Lipid rafts were isolated from NK92-C1 cells with stable SAP knockdown after cross-linking of 2B4 with antibodies. Western blot analysis of 2B4 immunoprecipitated from rafts and soluble fractions revealed no difference in stimulation-dependent raft recruitment of $2 \mathrm{~B} 4$ between knockdown and control cells
(Figures 3A,B). This finding indicates that SAP is not necessary for the raft recruitment of $2 \mathrm{~B} 4$.

\section{PHOSPHORYLATION OF 2B4 AND NTB-A IS INDEPENDENT OF SAP}

After recruitment to lipid rafts the ITSMs of 2B4 become phosphorylated (Watzl and Long, 2003). To analyze 2B4 phosphorylation in SAP knockdown cell lines, cells were stimulated by mixing with target cells expressing CD48. We found no difference in the induction of 2B4 phosphorylation between knockdown and control cells in NK92-C1 and YTS (Figures 3C,D). We also analyzed phosphorylation of NTB-A in NK92-C1 cells (Figure 3E). After stimulation with target cells expressing NTB-A we observed a comparable increase of NTB-A phosphorylation in SAP knockdown and control cells. These findings demonstrate that SAP is not necessary for the induction of $2 \mathrm{~B} 4$ and NTB-A phosphorylation.

The immunoprecipitates were also analyzed for coprecipitated adapter molecules. While stimulation of 2B4 led to recruitment of SAP in control cells, we detected no stimulation-dependent association of SAP with 2B4 in SAP knockdown cells (Figures 3C,D), although the knockdown cells still had residual amounts of SAP (Figure 2 and data not shown).

The amount of 2B4-bound EAT-2 increased with elevated phosphorylation after stimulation in control NK92-C1 cells (Figure 3C). However, in cells with reduced SAP expression EAT-2 could not be detected in the immunoprecipitates despite its unchanged expression (Figure 2A).

In non-stimulated NK92-C1 cells SAP and EAT-2 could be found in association with NTB-A (Figure 3E). However, while engagement of NTB-A increased the recruitment of both adapter proteins, we could neither detect SAP nor EAT-2 in immunoprecipitates from stimulated SAP knockdown cells (Figure 3E). These results reveal an unknown cooperativity between these

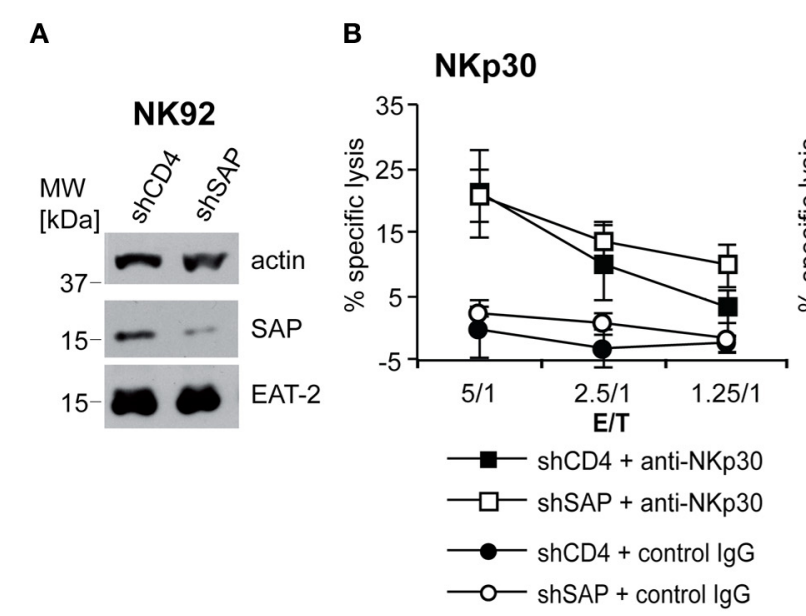

FIGURE 2 | SAP knockdown leads to diminished 2B4 or NTB-A-mediated cytotoxicity in cell lines. To study the impact of a SAP-knockdown on NK cell cytotoxicity, NK92-C1 cells stably expressing a small hairpin RNA against SAP (shSAP) or CD4 (shCD4) mRNA (as negative control) were analyzed.

(A) Western blot analysis of SAP and EAT-2 expression in NK92-C1 shCD4 and shSAP cells. (B) NK92-C1 shCD4 and shSAP were used as effector cells
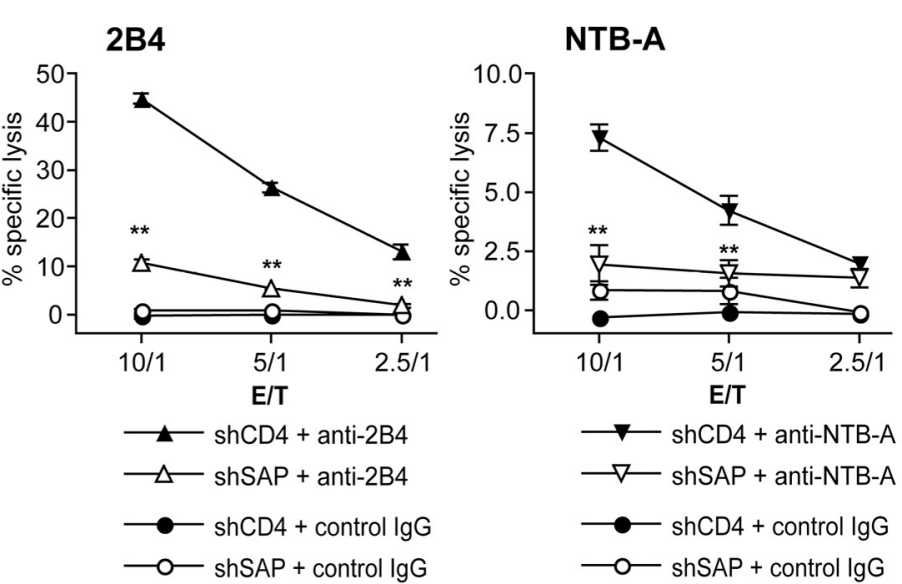

in a redirected ${ }^{51} \mathrm{Cr}$-release assay against $\mathrm{P} 815$ cells in the presence of IgG control Ab or antibodies against the receptors NKp30, 2B4, or NTB-A at different $E / T$ ratios. Data is shown as mean \pm SD of triplicates. Statistical significance of the reduced lysis by NK92-C1 shSAP compared to shCD4 cells was calculated using One-Way ANOVA and Dunnett's post test $\left({ }^{* *}\right.$ indicates $p<0.0001$ ). The results are from one representative experiment out of four. 


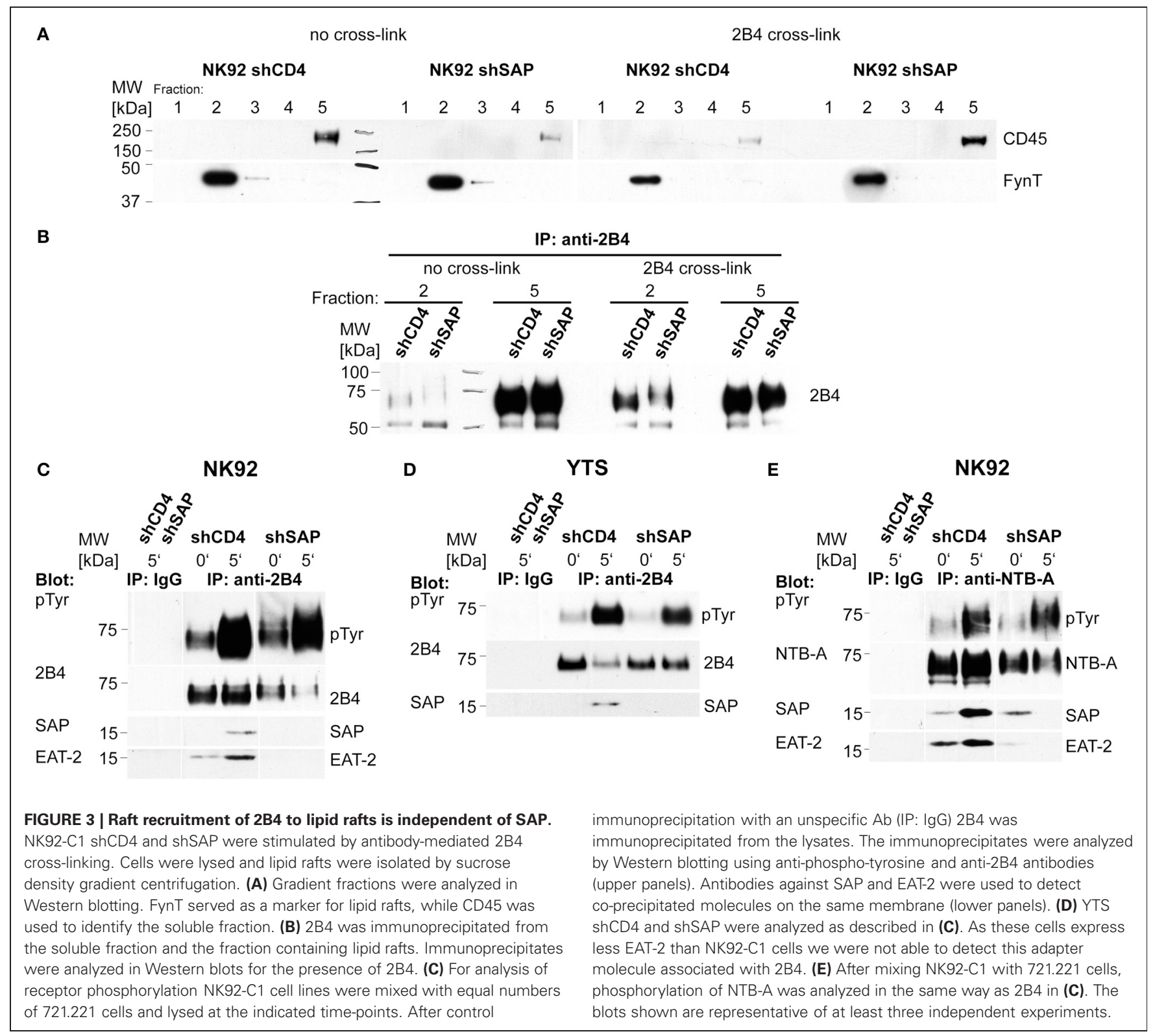

adapters and indicate that SAP facilitates the binding of EAT-2 to phosphorylated 2B4 and NTB-A.

\section{DISCUSSION}

In this study we used RNAi-mediated knockdown of SAP to investigate the molecular basis of the NK cell defect in XLP and to elucidate the role of this adapter molecule in the early events of SRR signaling. Our results show that the removal of functional SAP in in vitro propagated, IL-2 activated NK cell, or human NK cell lines is sufficient to generate the XLP phenotype.

While knockdown of SAP impaired cytotoxicity triggered by $2 \mathrm{~B} 4$ and NTB-A, we could not see any difference in the early signaling events of these two receptors, i.e., raft recruitment and receptor phosphorylation. This suggests that the impairment of signaling is due to defective recruitment of
FynT to phosphorylated receptors, which is essential to mediate down-stream signaling events (Bloch-Queyrat et al., 2005). Our finding is in contrast to a report of absent 2B4 phosphorylation in a study on NK cells from $\mathrm{SAP}^{-/-}$mice (Bloch-Queyrat et al., 2005). While we cannot exclude an off-target effect of our RNAi approach which could contribute to this difference, a recent study using murine NK cells came to the same conclusion as our study: Dong et al. found similar levels of phosphorylated 2B4 after receptor cross-linking in NK cells from $\mathrm{SAP}^{-/-}$and wild-type mice, as well as in mice transgenic for the SAP mutant R78A that cannot recruit FynT (Dong et al., 2012).

Our data also demonstrate that SAP is necessary for the binding of EAT-2 to $2 \mathrm{~B} 4$ and NTB-A. This novel cooperativity between these adapters suggests that the absence of SAP will also disrupt the unknown signaling pathways induced by EAT- 2 . 
These pathways do not seem to be necessary for a cytotoxic response, as we did not find a reduction of 2B4 or NTB-Amediated cytotoxicity after EAT-2 knockdown. This is in contrast to observations made with mouse NK cells. Cytotoxicity in NK cells from EAT-2 ${ }^{-/-}$mice has been reported to be either reduced (Wang et al., 2010) or enhanced (Roncagalli et al., 2005) depending on the strain tested. This could be due to the difference between species or to the difference in experimental approach. It could be possible that EAT-2 plays a role during NK cell development that has an impact on cytotoxicity. In this case NK cells from a knockout animal would show a difference, while a knockdown in mature cells shortly before cytotoxicity is tested would not affect the response. To answer this question a deeper knowledge of the EAT-2-mediated signaling pathways will be necessary.

\section{MATERIALS AND METHODS ANTIBODIES AND REAGENTS}

The following antibodies were used in this study: IgG1 control Ab MOPC21 (Sigma-Aldrich), anti-2B4 clone C1.7 (Immunotech), anti-NKG2D (R\&D Systems), anti-CD45 clone 69/CD45, and anti-Fyn clone 25 (both BD Transduction Laboratories), antiphospho-tyrosine clone 4G10 (upstate), polyclonal rabbit antiactin Ab (Sigma-Aldrich). The mouse monoclonal antibodies anti-NTB-A clone NT-7, anti-SAP clone SAP 23.1.5, anti-NKp30 clone p30-15, and the rabbit polyclonal antibodies anti-2B4, anti-NTB-A, and anti-EAT-2 have been generated in our lab as described previously (Watzl and Long, 2003; Flaig et al., 2004; Eissmann et al., 2005; Eissmann and Watzl, 2006; Byrd et al., 2007). The goat-anti-mouse-IgG Ab used for cross-linking was from Jackson ImmunoResearch Laboratories.

\section{CELLS}

Peripheral blood mononuclear cells (PBMC) were isolated from buffy coats or whole blood using density centrifugation over lymphocyte separation medium (PAA, Pasching, Germany). Polyclonal NK cells were purified from PBMC by negative selection using a NK cell negative isolation kit (Invitrogen). NK cells were between $90 \%$ and $99 \% \mathrm{NKp} 46^{+}, \mathrm{CD}^{-}$, and $\mathrm{CD}^{+} 6^{+}$. Cells were resuspended in IMDM with 10\% human serum (PromoCell, Heidelberg, Germany), penicillin/streptomycin containing 200 IU/mL IL-2 (National Institutes of Health cytokine repository), $1 \mu \mathrm{g} / \mathrm{mL}$ PHA-P and $5 \mathrm{ng} / \mathrm{mL}$ recombinant human IL-15 (R\&D Systems), mixed with irradiated JY cells $\left(5 \times 10^{5}\right.$ cells $\left./ \mathrm{mL}\right)$, and plated in 96-well round bottom plates.

The following cell lines were used in this study: the EBV-transformed, human B cell line 721.221, the murine

\section{REFERENCES}

Bloch-Queyrat, C., Fondaneche, M. C., Chen, R., Yin, L., Relouzat, F., Veillette, A., et al. (2005). Regulation of natural cytotoxicity by the adaptor SAP and the Src-related kinase Fyn. J. Exp. Med. 202, 181-192.

Bottino, C., Falco, M., Parolini, S., Marcenaro, E., Augugliaro, R., Sivori, S., et al. (2001). NTB-A [correction of GNTB-A], a novel SH2D1A-associated surface molecule contributing to the inability of natural killer cells to kill Epstein-Barr virus-infected B cells in X-linked lymphoproliferative disease. J. Exp. Med. 194, 235-246.

Bouchon, A., Cella, M., Grierson, H. L., Cohen, J. I., and Colonna, M. (2001). Activation of NK

mastocytoma line P815 (both grown in IMDM, 10\% FCS, penicillin/streptomycin), cells of the murine pre-B cell line BA/F3 stably transduced with the retroviral vector pBABE containing GFP, human CD48, or human NTB-A Y123F (Eissmann and Watzl, 2006) (all grown in RPMI 1640, 10\% FCS, $50 \mu \mathrm{M}$ 2-mercaptoethanol, penicillin/streptomycin, and selected with puromycin), stable CD4 or SAP knockdown lines generated from the cell lines NK92-C1 (grown in Alpha MEM, 12.5\% FCS, $12.5 \%$ donor horse serum, $50 \mu \mathrm{M}$ 2-mercaptoethanol, and penicillin/streptomycin), and YTS (grown in IMDM, 12.5\% FCS, $50 \mu \mathrm{M}$ 2-mercaptoethanol, and penicillin/streptomycin) by transduction with the retroviral vector pSHAG-MAGIC2 (Biocat) (Eissmann and Watzl, 2006).

\section{TRANSFECTION OF PRIMARY HUMAN NK CELLS}

Primary NK cells were transfected after 1 week of culture using the nucleofection method (Lonza). The following siRNAs were used: non-targeting siRNA \#1 (Thermo Scientific), Hs_SH2D1A_3 for SAP knockdown, and Hs_SH2D1B_1 for EAT-2 knockdown (both from Qiagen). 2-3 $\times 10^{6}$ cells per sample were transfected with 1 pmol of siRNA using the Nucleofector solution for human macrophages and the nucleofection program X-001, following the manufacturer's instructions at all other steps.

\section{CYTOTOXICITY ASSAYS, ANTIBODY CROSS-LINKING, CELL MIXING, AND RAFT ISOLATION}

Cytotoxicity ${ }^{51} \mathrm{Cr}$ release assays, antibody-mediated cross-linking, raft isolation, cell mixing, and western blot analysis were performed as described previously (Watzl and Long, 2003; Eissmann and Watzl, 2006). For immunoprecipitation of 2B4 from raft fractions $0.5 \mathrm{~mL}$ from the respective fractions were diluted with an equal volume of lysis buffer containing $0.5 \%$ Triton X-100 and incubated for $1 \mathrm{~h}$ at $4^{\circ} \mathrm{C}$ with $0.8 \mu \mathrm{g} \mathrm{C} 1.7 \mathrm{Ab}$ coupled to Dynabeads protein G (Invitrogen). The beads were then treated as described for the immunoprecipitation from cell lysates (Eissmann and Watzl, 2006). Western blotting signals were quantified using a densitometer (GS800, BioRad).

\section{STATISTICAL ANALYSIS}

Statistical analysis was performed using Prism 4.0 (GraphPad Software Inc.).

\section{ACKNOWLEDGMENTS}

The authors thank Birgitta Messmer for isolation and cultivation of primary NK cells, and Maren Claus for critically reading the manuscript. This work was funded by the DFG (SFB405 and WA 1552/5-1) and the BioFuture program of the BMBF.

cell-mediated cytotoxicity by a SAPindependent receptor of the $\mathrm{CD} 2$ family. J. Immunol. 167, 5517-5521. Byrd, A., Hoffmann, S. C., Jarahian, M., Momburg, F., and Watzl, C. (2007). Expression analysis of the ligands for the Natural Killer cell receptors NKp30 and NKp44. PLoS ONE 2:e1339. doi: 10.1371/ journal.pone.0001339
Chen, R., Relouzat, F., Roncagalli, R., Aoukaty, A., Tan, R., Latour, S., et al. (2004). Molecular dissection of 2B4 signaling: implications for signal transduction by SLAMrelated receptors. Mol. Cell. Biol. 24, 5144-5156.

Claus, M., Meinke, S., Bhat, R., and Watzl, C. (2008). Regulation of NK cell activity by 2B4, NTB-A 
and CRACC. Front. Biosci. 13, 956-965.

Dong, Z., Davidson, D., PerezQuintero, L. A., Kurosaki, T., Swat, W., and Veillette, A. (2012). The adaptor SAP controls NK cell activation by regulating the enzymes Vav-1 and SHIP-1 and by enhancing conjugates with target cells. Immunity 36, 974-985.

Eissmann, P., Beauchamp, L., Wooters, J., Tilton, J. C., Long, E. O., and Watzl, C. (2005). Molecular basis for positive and negative signaling by the natural killer cell receptor 2B4 (CD244). Blood 105, 4722-4729.

Eissmann, P., and Watzl, C. (2006). Molecular analysis of NTB-A signaling: a role for EAT-2 in NTB-A-mediated activation of human NK cells. J. Immunol. 177, 3170-3177.

Flaig, R. M., Stark, S., and Watzl, C. (2004). Cutting edge: NTB-A activates NK cells via homophilic interaction. J. Immunol. 172, 6524-6527.

Lanier, L. L. (2008). Up on the tightrope: natural killer cell activation and inhibition. Nat. Immunol. 9, 495-502.

Ma, C. S., Nichols, K. E., and Tangye, S. G. (2007). Regulation of cellular and humoral immune responses by the SLAM and SAP families of molecules. Annu. Rev. Immunol. 25, 337-379.

Morra, M., Lu, J., Poy, F., Martin, M., Sayos, J., Calpe, S., et al. (2001). Structural basis for the interaction of the free $\mathrm{SH} 2$ domain EAT-2 with SLAM receptors in hematopoietic cells. EMBO J. 20, 5840-5852.

Parolini, S., Bottino, C., Falco, M., Augugliaro, R., Giliani, S., Franceschini, R., et al. (2000). $\mathrm{X}$-linked lymphoproliferative disease. 2B4 molecules displaying inhibitory rather than activating function are responsible for the inability of natural killer cells to kill Epstein-Barr virus-infected cells. J. Exp. Med. 192, 337-346.

Roncagalli, R., Taylor, J. E., Zhang, S., Shi, X., Chen, R., Cruz-Munoz, M. E., et al. (2005). Negative regulation of natural killer cell function by EAT-2, a SAP-related adaptor. Nat. Immunol. 6, 1002-1010.
Schwartzberg, P. L., Mueller, K. L., Qi, H., and Cannons, J. L. (2009). SLAM receptors and SAP influence lymphocyte interactions, development and function. Nat. Rev. Immunol. 9, 39-46.

Tangye, S. G., Lazetic, S., Woollatt, E., Sutherland, G. R., Lanier, L. L., and Phillips, J. H. (1999). Cutting edge: human $2 \mathrm{~B} 4$, an activating NK cell receptor, recruits the protein tyrosine phosphatase SHP-2 and the adaptor signaling protein SAP. J. Immunol. 162, 6981-6985.

Wang, N., Calpe, S., Westcott, J., Castro, W., Ma, C., Engel, P., et al. (2010). Cutting edge: the adapters EAT-2A and $-2 \mathrm{~B}$ are positive regulators of CD244- and CD84-dependent NK cell functions in the C57BL/6 mouse. J. Immunol. 185, 5683-5687.

Watzl, C., and Long, E. O. (2003). Natural killer cell inhibitory receptors block actin cytoskeletondependent recruitment of 2B4 (CD244) to lipid rafts. J. Exp. Med. 197, 77-85.

Watzl, C., Stebbins, C. C., and Long, E. O. (2000). NK cell inhibitory receptors prevent tyrosine phosphorylation of the activation receptor
2B4 (CD244). J. Immunol. 165, 3545-3548.

Conflict of Interest Statement: The authors declare that the research was conducted in the absence of any commercial or financial relationships that could be construed as a potential conflict of interest.

Received: 15 November 2012; accepted: 03 January 2013; published online: 22 January 2013.

Citation: Meinke S and Watzl C (2013)

$N K$ cell cytotoxicity mediated by $2 B 4$ and NTB-A is dependent on SAP acting downstream of receptor phosphorylation. Front. Immun. 4:3. doi: 10.3389/fimmu. 2013.00003

This article was submitted to Frontiers in NK Cell Biology, a specialty of Frontiers in Immunology.

Copyright (c) 2013 Meinke and Watzl. This is an open-access article distributed under the terms of the Creative Commons Attribution License, which permits use, distribution and reproduction in other forums, provided the original authors and source are credited and subject to any copyright notices concerning any third-party graphics etc. 